\title{
Thermal Diffusion Separation of Petroleum Diamondoids and Protodiamondoids
}

\author{
G. A. Gadzhiev ${ }^{a}$, Ch. M. Badmaev ${ }^{b}$, G. N. Gordadze ${ }^{a, *}$, and M. V. Giruts ${ }^{a}$ \\ ${ }^{a}$ Gubkin Russian State University of Oil and Gas (National Research University), Moscow, 119991 Russia \\ ${ }^{b}$ Gorodovikov Kalmyk State University, Elista, 358000 Russia \\ *e-mail: gordadze@rambler.ru
}

Received October 7, 2020; revised October 19, 2020; accepted October 19, 2020

\begin{abstract}
Diamondoids were separated from protodiamondoids (precursors of diamondoids) in a saturated fraction $\left(150-350^{\circ} \mathrm{C}\right)$ of crude oil by thermal diffusion. It was found that in the course of thermal diffusion separation, $\mathrm{C}_{10}-\mathrm{C}_{13}$ adamantanes (tricyclo[3,3,1,13,7]decanes) and $\mathrm{C}_{14}-\mathrm{C}_{16}$ diamantanes (pentacyclo[7,3,1,1,12, $\left.0^{2,7}, 0^{6,11}\right]$ tetradecanes) are separated from the corresponding protoadamantanes and protodiamantanes and descend to the lower sections of the thermal diffusion column. In contrast to adamantanes and diamantanes, $\mathrm{C}_{18}-\mathrm{C}_{19}$ triamantanes (heptacyclo[7,7,1,13,15, $\left.0^{1,12}, 0^{2,7}, 0^{4,13}, 0^{6,11}\right]$ octadecanes) are not separated from prototriamantanes and are concentrated in the lower sections of the thermal diffusion column. Catalytic isomerization of this thermal diffusion fraction with aluminum bromide was carried out to further confirm that the numerous compounds that have the same characteristic ions as triamantanes but elute later are polycyclic (most likely bridged) hydrocarbons, specifically prototriamantanes. As a result, all these polycyclic compounds were isomerized into triamantanes. Similarly, in the course of isomerization of thermal diffusion fractions containing protoadamantanes and protodiamantanes, all of these turned into adamantanes and diamantanes, respectively.
\end{abstract}

Keywords: diamond-like hydrocarbons, adamantane, diamantane, triamantane, thermal diffusion

DOI: $10.1134 / \mathrm{S} 0965544121020110$

Diamond-like hydrocarbons, such as adamantanes (tricyclo[3,3,1, $\left.1^{3,7}\right]$ decanes), diamantanes (pentacyclo[7,3,1,1 $\left.1^{4,12}, 0^{2,7}, 0^{6,11}\right]$ tetradecanes), triamantanes (heptacyclo[7,7,1,1 $\left.1^{3,15}, 0^{1,12}, 0^{2,7}, 0^{4,13}, 0^{6,11}\right]$ octadecanes), etc., are of great practical importance, as they are promising synthons for fine organic synthesis, raw materials for nanochemistry, molecular building blocks of nanoscaled objects, etc. In particular, they are used as raw materials to produce valuable chemicals and materials with unique properties, including pharmaceuticals, biomarkers, heat-resistant polymers, 1-, 2-, and 3-dimensional nanomaterials, high-energy fuels, lubricants and additives, etc. [1-5].

However, broad-scale application of diamond-like hydrocarbons is limited, on the one hand, by the difficulty of their synthetic production (especially regarding highmolecular members of the series) and, on the other hand, by their extremely low concentrations in crude oils and condensates, which are their only natural source. Moreover, our prior studies $[5,6]$ revealed only tiny amounts of these hydrocarbons in the absolute majority of crude oils and condensates. As such, their relative concentration in offshore crude oils is substantially lower than in onshore crudes, due to the presence of the socalled "protodiamondoids" (precursors of adamantanes). In other words, many peaks of other hydrocarbons elute in mass chromatograms with characteristic ions of $\mathrm{C}_{10}-\mathrm{C}_{13}$ adamantanes, $\mathrm{C}_{14}-\mathrm{C}_{15}$ diamantanes, and $\mathrm{C}_{18}-\mathrm{C}_{19}$ triamantanes $(\mathrm{m} / \mathrm{z} 135,136,149,163 ; \mathrm{m} / \mathrm{z} 187,188,201$, and $m / z 239,240$, respectively).

Despite their small amounts in crude oils and condensates, we find it relevant to study the feasibility of the isolation of the abovementioned hydrocarbons in pure form from natural objects, in particular from crude oils and gas condensates.

Certain attempts have been made to isolate diamondoids from crude oils by physical methods such as complexing with urea, extraction with various solvents, adsorbentbased methods, or preparative gas-liquid chromatography [7-15]. However, to the authors' knowledge, there are no 
available references on the regularities of the separation of diamondoids and protodiamondoids by thermal diffusion (TD), although this method was previously known to be rather effective in separation of $n$-alkanes, iso-alkanes, mono-, bi-, tri-, and tetracyclic saturated hydrocarbons $[16,17]$. Therefore, this study was intended to identify the potential for thermal diffusion separation of diamondoids from protodiamondoids.

\section{EXPERIMENTAL}

We sampled crude oil from well no. 406 in the Kurgannoye oil field (sample interval 2012-2015 m, Lower Cretaceous Aptian, $0.8339 \mathrm{~g} / \mathrm{cm}^{3}$ density at $20^{\circ} \mathrm{C}$ ). The diamondoid content in the crude oil was about $0.03 \%$, while the protodiamondoid content amounted to about an order of magnitude higher. A $150-350^{\circ} \mathrm{C}$ fraction of the crude oil was drawn off ( $44 \%$ of crude oil) and dearomatized over silica gel prior to TD separation.

A TD column of the most widespread Melpolder type was used to separate protodiamondoids from diamondoids [18]. Any thermal diffusion apparatus consists of two surfaces, one of which is heated and the other is cooled. A required temperature gradient is established by varying the surface temperatures. The separation capacity of a TD column is inversely proportional to the biquadrate of the gap width (space between the surfaces) and directly proportional to the gap length. The effective gap width is usually 0.25 to $3.00 \mathrm{~mm}$, and the column loading capacity is 3 to $400 \mathrm{~mL}$, depending on the type. The most common column type is vertical double-tube (hollow-gapped, packed, or coiled). In such columns a convection current rises along the hot wall and descends along the cold wall. Molecules diffusing to the hot wall rise across the column under the effect of the current. A Melpolder column consists of two concentric tubes with a $0.25 \mathrm{~mm}$ gap [18]. Outlets for withdrawal of the fractions are spaced at equal intervals. In our case the column contained seven outlets equipped with taps and spaced at about $20 \mathrm{~cm}$. The lowest section was drawn off from the last tap (no. 7), and the uppermost section from tap no. 2. We experimentally found the temperature gradient between the walls to be at least 120 deg to ensure the fractional separation. Therefore, the external wall was maintained at $130^{\circ} \mathrm{C}$ and the water-cooled internal wall at $10^{\circ} \mathrm{C}$. The $150-350^{\circ} \mathrm{C}$ fraction was initially charged into the TD column and, after $80 \mathrm{~h}$ of a continuous experiment run, the TD separation products were unloaded successively, starting from the upper tap (no. 2). Each TD fraction had a volume of about $5 \mathrm{~mL}$.

Both the separated TD fractions and the initial fraction were tested by chromatography-mass spectrometry (GC/MS) using characteristic ions $m / z$ 135, 136, 149, and 163 for $\mathrm{C}_{10}-\mathrm{C}_{13}$ adamantanes and protoadamantanes, $m / z$ 187, 188, and 201 for $\mathrm{C}_{14}-\mathrm{C}_{16}$ diamantanes and protodiamantanes, and $m / z 239$ and 240 for $\mathrm{C}_{18}-\mathrm{C}_{19}$ triamantanes and protodiamantanes. The GC/MS was performed on an Agilent 6890N/5975C instrument. The temperature was programmed from 70 to $290^{\circ} \mathrm{C}$ with a ramp rate of $4 \mathrm{deg} / \mathrm{min}$. The hydrocarbons were separated on $25 \mathrm{~m} \times 0.25 \mathrm{~mm} \times 0.5 \mu \mathrm{m}$ capillary columns with a HP-1MS stationary phase and with helium as a carrier gas. All spectra were recorded at an ionization energy of $70 \mathrm{eV}$ and an accelerating voltage of $3500 \mathrm{~V}$. The ionization chamber temperature was $250^{\circ} \mathrm{C}$.

The compounds were identified by adding putative reference compounds to the tested samples and by using the NIST 2.0 mass spectra library.

\section{RESULTS AND DISCUSSION}

Figures 1-3 demonstrate the mass chromatograms of the initial saturated fraction $\left(150-350^{\circ} \mathrm{C}\right)$ and of the TD fractions taken from the middle (tap no. 4) and from the lowest section (tap no. 7) of the TD column. The fraction taken from section 4 of the TD column was chosen as an illustrative example because it had an about average (between the top and bottom of the column) hydrocarbon distribution.

Adamantanes and protoadamantanes were distributed across the height of the TD column as clearly shown in Fig. 1.

For example, while the TD fraction drawn off from section 4 contains only protoadamantanes, the TD fraction from the lowest ( 7 th) section protoadamantanes are nearly completely absent (see Fig. 1). As noted above, the hydrocarbon distribution in section 4 represents the average between the top and bottom. Indeed, sections 2 and 3 , like section 4 , contain protoadamantanes alone, while adamantanes are present along with protoadamantanes in the lower sections 5 and 6 , and the relative content of adamantanes increases as we move towards the TD column bottom.

A similar pattern was observed in the diamantane case (Fig. 2). It is clearly seen that the fraction from section 4 (like those from sections 2 and 3 ) of the TD column lacks diamantanes, methyl diamantanes, and dimethyl diaman-

PETROLEUM CHEMISTRY Vol. 61 No. 22021 
(a)

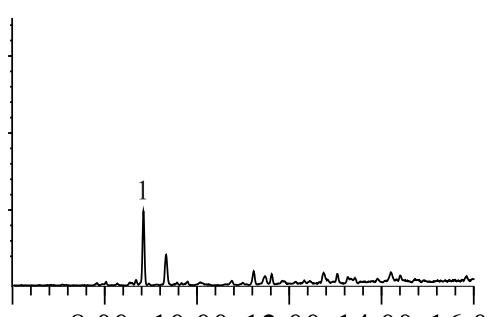

$8.00 \quad 10.0012 .0014 .0016 .00$

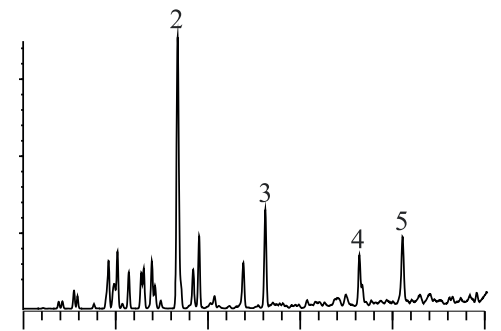

$8.00 \quad 10.0012 .0014 .0016 .00$
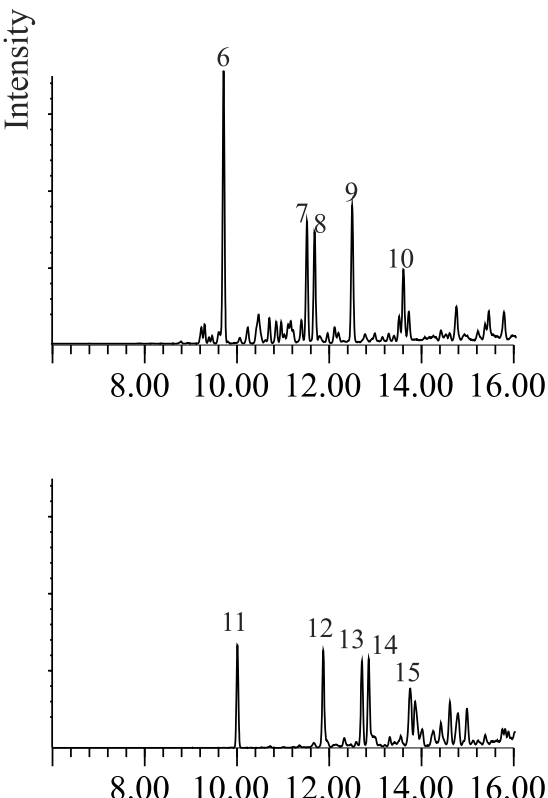

Time, $\min$ (b)
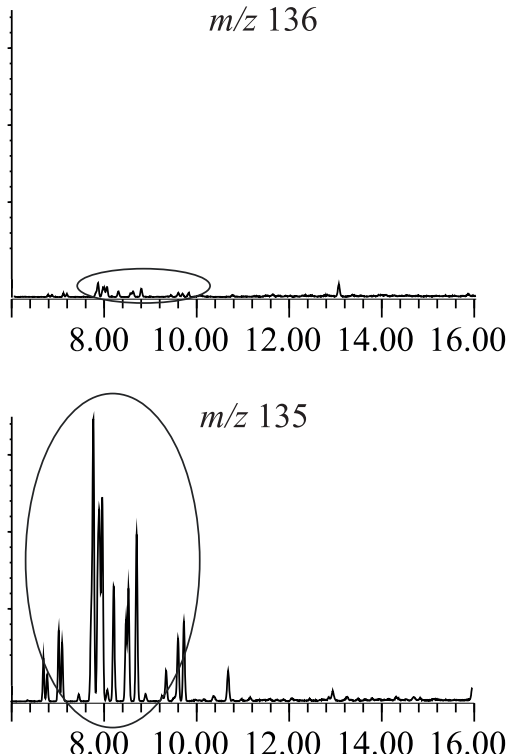

$m / z 149$
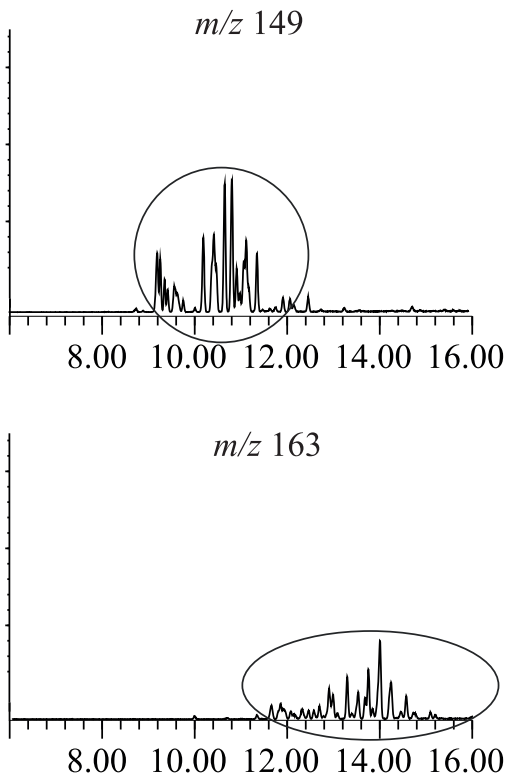

Time, $\min$ (c)

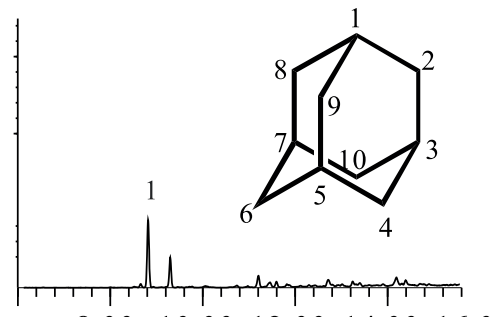

8.0010 .0012 .0014 .0016 .00
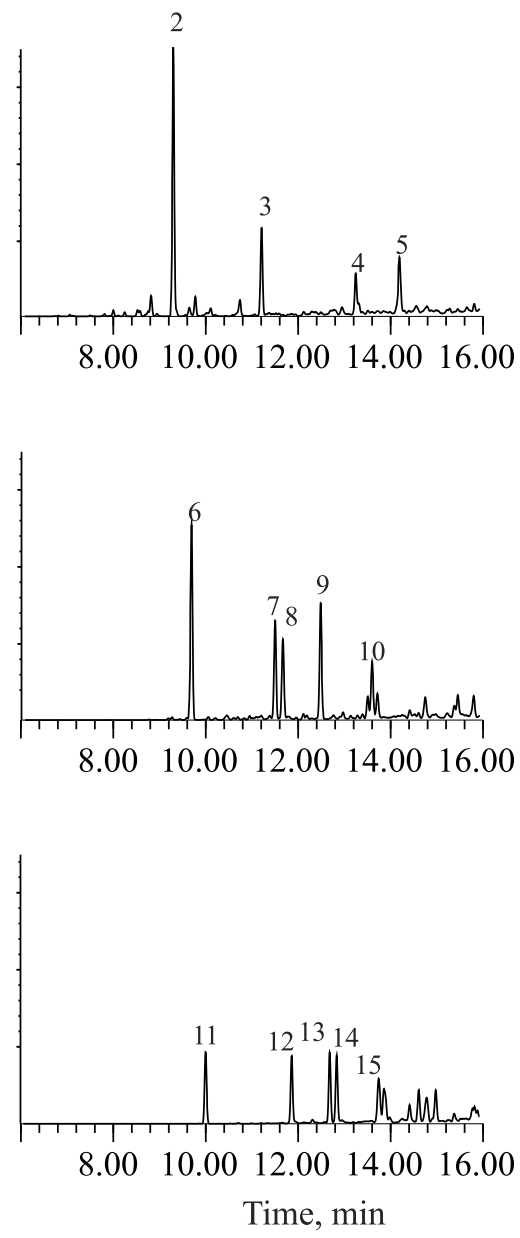

Fig. 1. Mass chromatograms of $\mathrm{C}_{10}-\mathrm{C}_{13}$ adamantanes in (a) the initial fraction $\left(150-350^{\circ} \mathrm{C}\right.$ ) and in the TD fractions taken from (b) section 4 and (c) section 7 (lowest section) of the TD column (taps no. 4 and no. 7, respectively): (1) adamantane; (2) 1-methyl adamantane; (3) 2-methyl adamantane; (4) 1-ethyl adamantane; (5) 2-ethyl adamantane; (6) 1,3-dimethyl adamantane; (7) cis-1,4-dimethyl adamantane; (8) trans-1,4-dimethyl adamantane; (9) 1,2-dimethyl adamantane; (10) 1-ethyl-3-methyl adamantane; (11) 1,3,5-trimethyl adamantane; (12) 1,3,6-trimethyl adamantane; (13) cis-1,3,4-trimethyl adamantane; (14) trans-1,3,4-trimethyl adamantane; (15) 1-ethyl-3,5-dimethyl adamantane. Protoadamantanes are circled.

tanes, and only contains protodiamantanes. On theotherhand, diamantanes, 4-, 1-, 3-methyl diamantanes, and dimethyl diamantanes are concentrated in the lowest section of the TD column (tap no. 7). However, in contrast to the protoadamantanes, which are absent in the lowest column section, a certain amount of protodiamantanes descend down the column along with diamantanes. This is because protodiamantanes contain more cycles than protoadamantanes.

A somewhat different pattern is observed for triamantanes, when compared with adamantanes or diamantanes. 
(a)
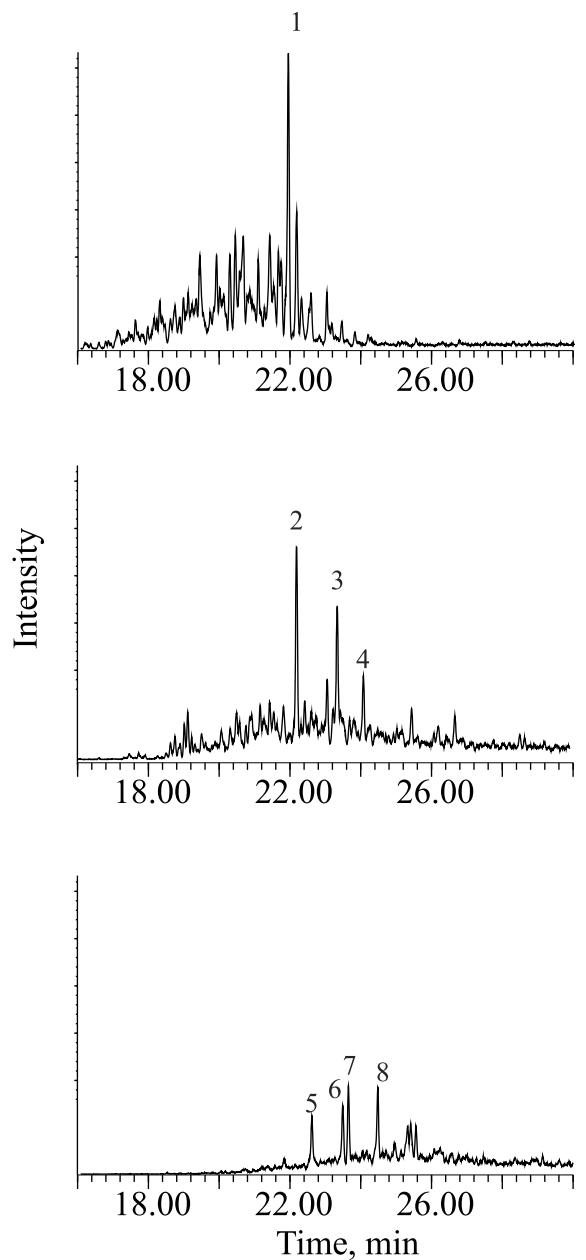

(b)
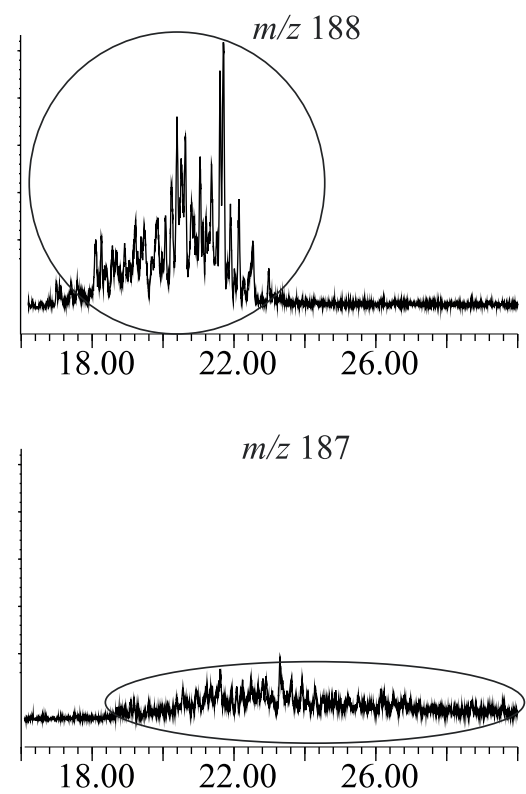

$m / z 201$

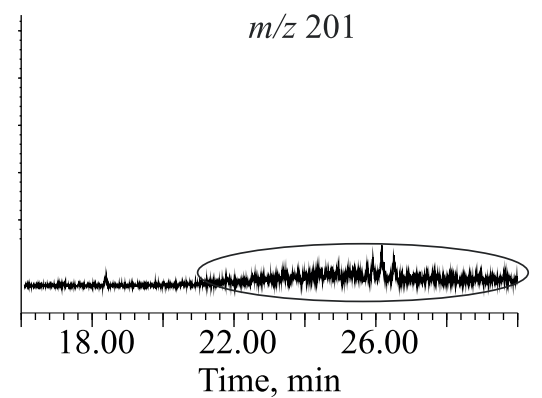

(c)
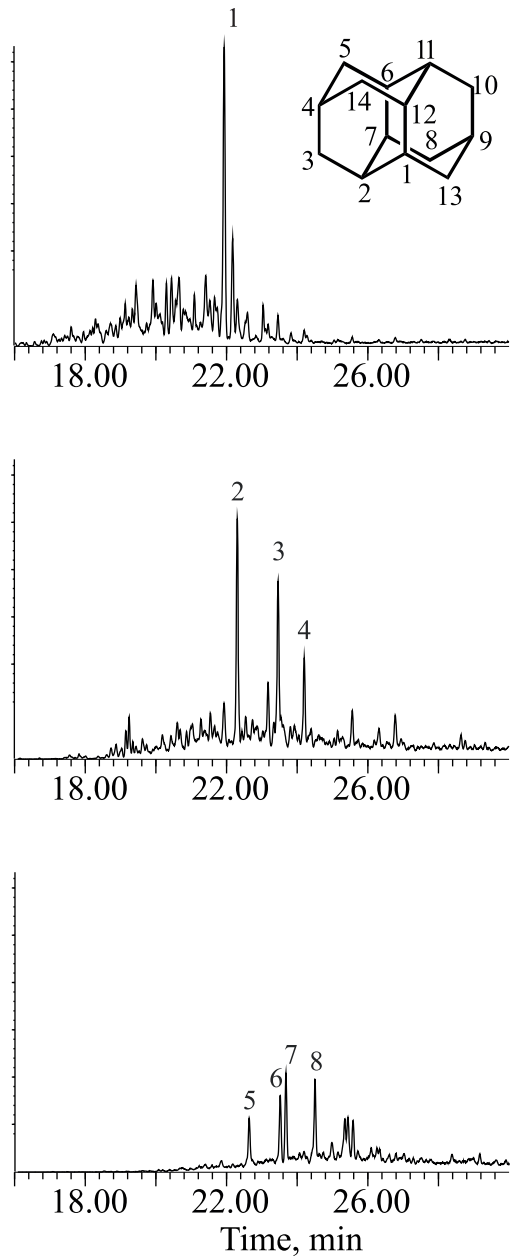

Fig. 2. Mass chromatograms of $\mathrm{C}_{14}-\mathrm{C}_{16}$ diamantanes in (a) the initial fraction $\left(150-350^{\circ} \mathrm{C}\right)$ and in the TD fractions taken from (b) section 4 and (c) section 7 (lowest section) of the TD column (taps no. 4 and no. 7, respectively): (1) diamantane; (2) 4-methyl diamantane; (3) 1-methyl diamantane; (4) 3-methyl diamantane; (5) 4,9-dimethyl diamantane; (6) 1,4- and 2,4-dimethyl diamantanes; (7) 4,8-dimethyl diamantane; (8) 3,4-dimethyl diamantane. Protoadamantanes are circled.

Triamantanes are also concentrated in the lowest (7th) TD fraction (see Fig. 3).

It is worth noting that, along with triamantanes, a group of peaks that elute later also drops towards the TD column bottom. Obviously, these peaks belong to polycyclic hydrocarbons, most likely bridged compounds. Moreover, unlike protodiamantanes, all of these hydrocarbons are concentrated in the column bottom. It should be noted that the same group of peaks was also present in the initial fraction. To verify that the peaks at $m / z 239$ and 240, which elute after triamantanes in the mass chromatograms, are actually their precursors, we carried out isomerization of hydrocarbons of the lowest TD fraction with aluminum bromide and found that all of these turn into triamantanes (Fig. 3d). In addition, we isomerized other TD fractions containing protoadamantanes and protodiamantanes
(Figs. $1 \mathrm{~b}$ and $2 \mathrm{~b}$ ), and they also turned into adamantanes and diamantanes in the presence of aluminum bromide.

\section{AUTHOR INFORMATION}

G.A. Gadzhiev, ORCID: https://orcid.org/0000-00028130-4801

Ch.M. Badmaev, ORCID: https://orcid.org/0000-00033569-3532

G.N. Gordadze, ORCID: https://orcid.org/0000-00025300-3059

M.V. Giruts, ORCID: https://orcid.org/0000-0001-50576532

\section{CONCLUSIONS}

In summary, it was demonstrated that, using thermal diffusion separation, adamantanes and diamantanes 
(a)

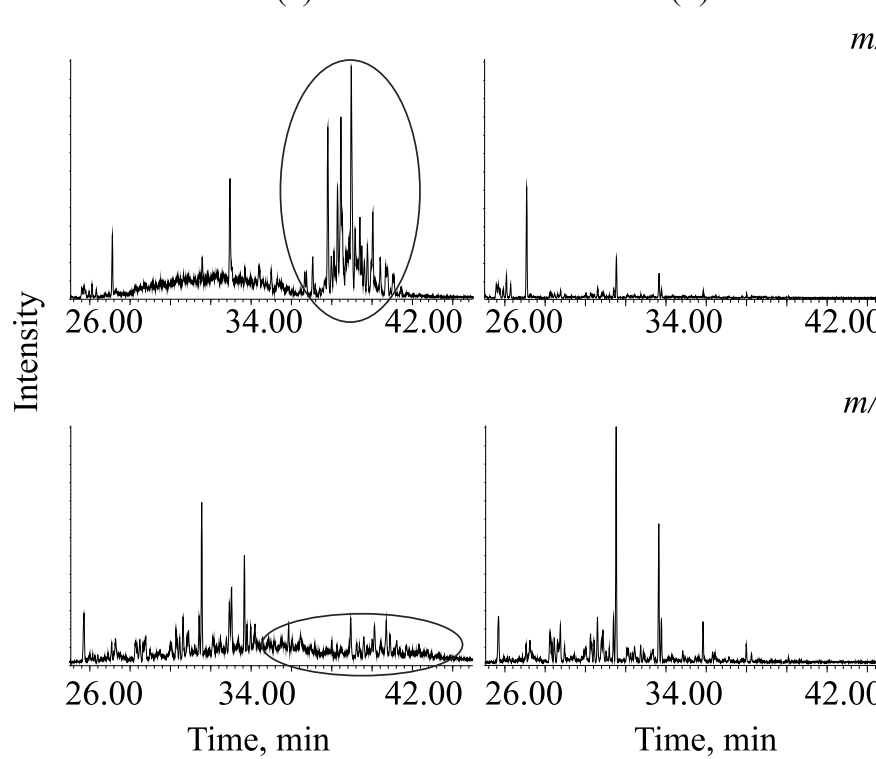

(c)

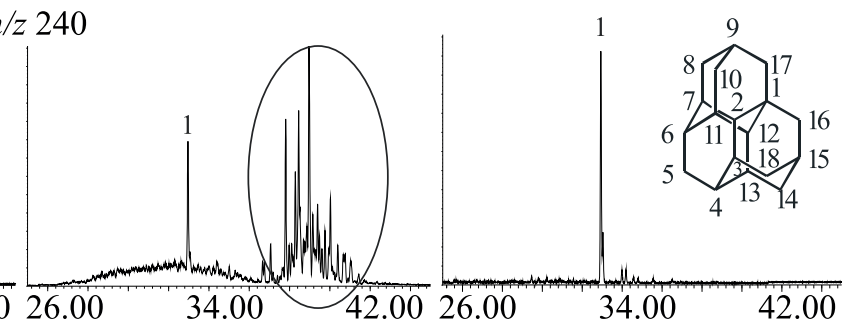

Fig. 3. Mass chromatograms of $\mathrm{C}_{18}-\mathrm{C}_{19}$ triamantanes in (a) the initial fraction $\left(150-350^{\circ} \mathrm{C}\right)$, in the TD fractions taken from (b) section 4 and (c) section 7 (lowest section) of the TD column (taps no. 4 and no. 7, respectively), and in (d) the products of the lowest TD fraction isomerization with aluminum bromide: (1) triamantane; (2) 9-methyl triamantane. Protoadamantanes are circled.

are separated from protoadamantanes and protodiamantanes and concentrated in the lower sections of the TD column. In contrast, triamantanes are not separated from prototriamantanes and are concentrated together in the lower sections of the TD column.

\section{CONFLICT OF INTEREST}

The authors declare no conflict of interest requiring disclosure in this article.

\section{OPEN ACCESS}

This article is distributed under the terms of the Creative Commons Attribution 4.0 International License (http:// creativecommons.org/licenses/by/4.0/), which permits unrestricted use, distribution, and reproduction in any medium, provided you give appropriate credit to the original author(s) and the source, provide a link to the Creative Commons license, and indicate if changes were made.

\section{REFERENCES}

1. Bagrii, E.I., Adamantany (Adamantanes), Moscow: Nauka, 1989.

2. Nekhaev, A.I., Bagrii, E.I., and Maximov, A.L., Petrol. Chem., 2011, vol. 51, no. 2, pp. 86-95. https://doi.org/10.1134/S0965544111020095

3. Mansoori, G.A., Diamondoid Molecules with Application in Biomedicine, Material Science, Nanotechnology, and Petroleum Science, Singapore: World Scientific Publishing Co. Pte. Ltd., 2012.

4. Bagrii, E.I. and Maravin, G.B., Petrol. Chem., 2013, vol. 53, no. 6 , pp. 418-422.

https://doi.org/10.1134/S0965544113060029
5. Giruts, M.V. and Gordadze, G.N., Khimiya i geokhimiya uglevodorodov almazopodobnogo stroeniya (Chemistry and Geochemistry of Diamond-like Hydrocarbons), Moscow: Nedra, 2017.

6. Gordadze, G.N., Uglevodorody v neftyanoi geokhimii. Teoriya $i$ praktika (Hydrocarbons in Petroleum Geochemistry. Theory and Practice), Moscow: Ross. Gos. Univ. Nefti i Gaza im. I.M. Gubkina, 2015.

7. Bagrii, E.I. Amosova, E.I., and Sanin, P.I., Neftekhim., 1966, vol. 6, no. 5, pp. 665-670.

8. Dahl, J.E. and Carlson, R.M., WO Patent 2002096804-A, 2003.

9. Alexander, R.A., Knight, C.E., and Whitehurst, D.D., US Patent 4982049, 1991.

10. Partridge, R.D. and Whitehurst, D.D., US Patent 5019665, 1991.

11. Swanson, D.S., US Patent 5461184, 1995.

12. Alexander, R.A., Knight, C.E., and Whitehurst, D.D., US Patent 4952747, 1990.

13. Alexander, R.A., Knight, C.E., and Whitehurst, D.D., US Patent 4952749, 1990.

14. Alexander, R.A., Knight, C.E., and Whitehurst, D.D., US Patent 4982049, 1991.

15. Rollmann, L.D., Green, L.A., Bradway, R.A., and Timken, H.K.C., Catal. Today, 1996, vol. 31, pp. 163-169. https://doi.org/10.1016/0920-5861(96)00021-1

16. Petrov, Al.A., Uglevodorody nefti (Petroleum Hydrocarbons), Moscow: Nauka, 1984.

17. Solodkov, V.K., Mikhnovskaya, A.A., Smirnov, B.A., and Petrov, Al.A., Neftekhim., 1969, vol. 9, no. 4, pp. 491-499.

18. Sovremennye metody issledovaniya neftei. Spravochnometodicheskoe posobie (Modern Methods of Oil Research. Reference and Methodological Manual), Bogomolov, A.I., Temyanko, M.B., and Khotyntseva, L.I., Eds., Leningrad: Nedra, 1984. 